\title{
ARTICLE
}

\section{Injection and controlled motion of conducting domain walls in improper ferroelectric $\mathrm{Cu}-\mathrm{Cl}$ boracite}

Raymond G.P. McQuaid ${ }^{1}$, Michael P. Campbell ${ }^{1}$, Roger W. Whatmore ${ }^{2}$, Amit Kumar $^{1}$ \& J. Marty Gregg ${ }^{1}$

Ferroelectric domain walls constitute a completely new class of sheet-like functional material. Moreover, since domain walls are generally writable, erasable and mobile, they could be useful in functionally agile devices: for example, creating and moving conducting walls could make or break electrical connections in new forms of reconfigurable nanocircuitry. However, significant challenges exist: site-specific injection and annihilation of planar walls, which show robust conductivity, has not been easy to achieve. Here, we report the observation, mechanical writing and controlled movement of charged conducting domain walls in the improper-ferroelectric $\mathrm{Cu}_{3} \mathrm{~B}_{7} \mathrm{O}_{13} \mathrm{Cl}$. Walls are straight, tens of microns long and exist as a consequence of elastic compatibility conditions between specific domain pairs. We show that site-specific injection of conducting walls of up to hundreds of microns in length can be achieved through locally applied point-stress and, once created, that they can be moved and repositioned using applied electric fields.

\footnotetext{
${ }^{1}$ Centre for Nanostructured Media, School of Mathematics and Physics, Queen's University Belfast, Belfast BT7 1NN, UK. ${ }^{2}$ Department of Materials, Imperial College London, Exhibition Road, London SW7 2AZ, UK. Correspondence and requests for materials should be addressed to J.M.G. (email: m.gregg@qub.ac.uk).
} 
dentifying and understanding the key factors that generate reliable, well-controlled conductivity along ferroic domain walls is a crucial step towards realizing prototype domain wall-based nanoelectronic devices ${ }^{1-6}$. In the original landmark observations of domain wall conductivity in thin-films of $\mathrm{BiFeO}_{3}$ (ref. 5) significant densities of charge-carrying point defects were seen to play a decisive role in determining wall transport ${ }^{6}$. This situation occurs readily in naturally leaky systems and can be created artificially by either extrinsic doping ${ }^{6,7}$, or by vacuum annealing, as demonstrated in thin-films of ferroelectric $\mathrm{Pb}(\mathrm{Zr}, \mathrm{Ti}) \mathrm{O}_{3}$ (ref. 8). This offers a large degree of flexibility in engineering domain wall transport but without strict defect control or quantification can lead to difficulties with consistency and reproducibility in conduction, such as is seen to be the case in $71^{\circ}$ walls in $\mathrm{BiFeO}_{3}$ (ref. 9).

The engineering of polar discontinuities across domain walls is an attractive alternative to the extrinsic manipulation of domain wall transport through sample processing. Conductivity anomalies have been rationalized as an intrinsic feature of these chargedtype domain walls, where the near-field dipolar configuration either side of the boundary is oriented head-to-head or tail-to-tail and orders of magnitude enhancement of local conductivity along charged domain walls have been reported ${ }^{10-12}$. However, in proper ferroelectrics, where polarization is the primary order parameter, such polar discontinuities usually are highly unstable and spontaneously forming charged-wall configurations are therefore rarely seen to occur ${ }^{13}$. The situation can be very different in improper ferroelectrics. These are materials where the primary order parameter is not polarization but instead is a physical quantity, such as spontaneous strain, which exhibits different transformation properties ${ }^{14}$. A crucial aspect of the classification is that the development of polarization is unable to fully account for the reduction in symmetry through the transition (and therefore cannot be the primary order parameter). The rare-earth manganites provide a textbook case, where development of the primary order parameter upon cooling through the transition, comprising of a structural tilting of $\mathrm{MnO}_{5}$ trigonal bipyramids (known as trimerization) ${ }^{15}$, also leads to a spontaneous polarization developing as a secondary order parameter. The mechanical compatibility of structural domain variants, which requires matching of the domain-related deformations at the boundary plane, as formalized by Fousek and Janovec ${ }^{16}$, is seen to take precedence over electrostatic near-field neutrality and enables proliferation of stable charged conducting domain walls ${ }^{2}$. A somewhat analogous case has been reported more recently with the observation of charged walls in improper ferroelectric-layered-perovskite $\left(\mathrm{Ca}, \mathrm{Sr}_{3}\right)_{3} \mathrm{Ti}_{2} \mathrm{O}_{7}$ (ref. 11). Here, rotations/tilting of $\mathrm{TiO}_{6}$ octahedra leads to a ferroelastic domain structure but also the concurrent development of a spontaneous polarization, due to an accompanying displacement of the $\mathrm{Ca} / \mathrm{Sr}$ ions. Like in manganites, mechanical compatibility between structural domain variants takes precedence over polar continuity, enabling spontaneous formation of charged type domain walls. However, the existence of improper ferroelectricity is not a sufficient criterion to ensure charged-wall formation. For example, in ferroelastic improper-ferroelectric $\mathrm{Gd}_{2}\left(\mathrm{MoO}_{4}\right)_{3}$, which undergoes a transition from paraelectric $\overline{4} 2 \mathrm{~m}$ to ferroelectric/ferroelastic $m m 2$ (ref. 17), only uncharged $180^{\circ}$ walls are expected to be stable ${ }^{18}$. To determine the charge or neutral state of the walls, one must examine the expected equilibrium domain wall configurations associated with the primary order parameter and identify the possible polar configurations at the wall, based on the explicit orientation relationship between the primary order parameter and polarization. Using the approach pioneered by Fousek and Janovec ${ }^{16}$, Erhart has carried out a comprehensive survey of permissible domain wall orientations for all ferroelastic/ferroelectric species based on mechanical compatibility considerations ${ }^{18}$ and lists cases where charged walls may be expected.

As it stands, improper ferroelectrics are promising candidates for exhibiting conducting domain walls with robust transport characteristics but so far they have not been successfully used to deliver site-specific wall deployment and positioning. For example, in the manganites the fact that the walls are curved and consist of irregular sections with enhanced transport makes it difficult to envisage how they can be easily manipulated in a device. As-grown improper-ferroelectric $(\mathrm{Ca}, \mathrm{Sr})_{3} \mathrm{Ti}_{2} \mathrm{O}_{7}$ crystals $^{11}$ have the advantage of exhibiting planar rectilinear conducting walls, but a means for their controlled injection and positioning has not been shown. Indeed, there is no evidence at present to suggest that they persist after thermal or electric field cycling. In contrast, techniques for controlling domain walls are betterestablished for conventional proper ferroelectrics. Local writing of conducting walls using a biased atomic force microscope probe has proved effective and popular for thin-films $s^{3,5,8,12}$. Even so, more device-relevant designs, such as that explored by Sluka and co-workers at EPFL ${ }^{10,19}$ in bulk $\mathrm{BaTiO}_{3}$ crystals, lack site-specific injection and control capabilities.

In this article we report site-specific injection and positional control of conducting charged domain walls in proper-ferroelastic improper-ferroelectric $\mathrm{Cu}_{3} \mathrm{~B}_{7} \mathrm{O}_{13} \mathrm{Cl}$ single crystals. In this system, mechanical compatibility conditions between specific domain pairs can lead to generation of a $90^{\circ}$ head-head/tail-tail polar configuration. Spontaneously formed charged boundaries are seen to be rectilinear and of order tens of microns in length. We show that local mechanical stress can be used for repeatable site-specific injection of conducting wall arrays that span up to hundreds of microns in length and then show how individual walls can be moved using applied planar electric fields. Such an approach may be useful in the design of piezoresistive-type devices $^{20}$, where functionality is derived from stress-induced conducting domain walls.

\section{Results}

Boracite crystal structure. The family of minerals commonly described as boracites have composition $\mathrm{M}_{3} \mathrm{~B}_{7} \mathrm{O}_{13} \mathrm{X}$, where $\mathrm{M}$ is a divalent metal and $\mathrm{X}$ is usually a halogen (but can also be $\mathrm{OH}^{-}$ or $\mathrm{S}^{2-}$ (ref. 21)). Boracites have a complex crystal structure with a large unit cell ${ }^{22}$ and undergo a ferroelastic transition from cubic point group $\overline{4} 3 m$ to orthorhombic $m m 2$ (ref. 23). The natural boracite $\left(\mathrm{Mg}_{3} \mathrm{~B}_{7} \mathrm{O}_{13} \mathrm{Cl}\right)$ mineral structure can be envisaged as a three dimensional unbroken $\mathrm{B}-\mathrm{O}$ network with $\mathrm{Mg}^{2}+$ and $\mathrm{Cl}^{-}$ ions filling the interstices, as illustrated in Fig. 1a,b (further details in Supplementary Fig. 1). The B-O network consists of cornersharing $\mathrm{BO}_{4}$ tetrahedra and $\mathrm{BO}_{3}$ triangles with each $\mathrm{Mg}^{2+}$ ion being octahedrally coordinated with four oxygens and two chlorines. Upon cooling through the transition, a structural shearing of the cubic unit cell occurs ${ }^{24}$ (Fig. 1c). The transition is improper ferroelectric in character: alternate layers of $\mathrm{Cl}^{-}$ions shift along different $\langle 111\rangle_{\text {pseudocubic (pc) }}$ directions leading to a net displacement of the centre of negative charge along $[001]_{\mathrm{pc}}$ while all $\mathrm{Mg}^{2+}$ ions shift along $\langle 100\rangle_{\mathrm{pc}}$ directions ${ }^{25}$. For the $\mathrm{Mg}^{2+}$ ions, two perpendicular sublattices can be identified where the ions perform antiparallel shifts and a third where the net displacement is along $[00 \overline{1}]_{\mathrm{pc}}$ (ref. 25). The net relative shifts of the halogen and metal ions along $[001]_{\mathrm{pc}}$ lead to the development of a dipole moment along this axis (and the possibility for six equivalent $\langle 100\rangle_{\mathrm{pc}}$ oriented polar variants upon symmetry breaking from the high-temperature cubic state).

Identification of charged walls and conductivity mapping. Local surface topography of a $\{100\}_{\mathrm{pc}}$-faced $\mathrm{Cu}-\mathrm{Cl}$ boracite single 
a

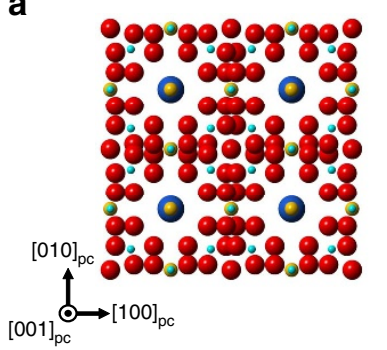

b

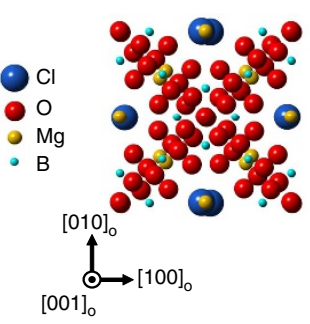

C

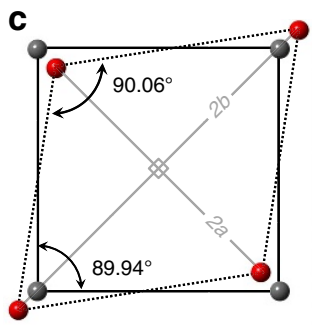

d

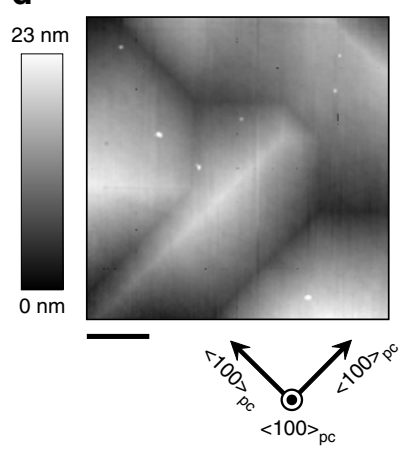

f

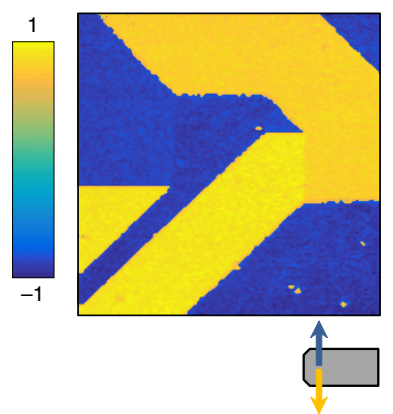

e
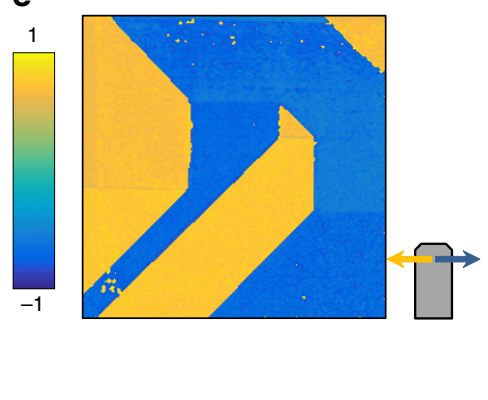

g

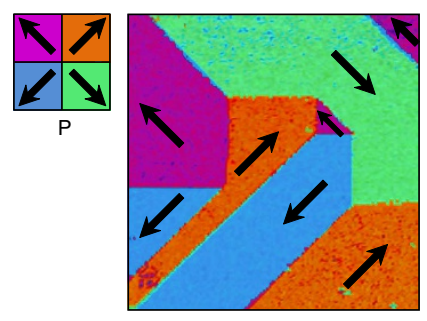

Figure 1 | Crystal structure and domains in boracites. Atomic structure of the $\mathrm{Mg}_{3} \mathrm{~B}_{7} \mathrm{O}_{13} \mathrm{Cl}$ unit cell projected on (001) cubic for the cubic phase (a) and (001) $)_{\text {pseudocubic }}$ for the orthorhombic phase (b). (c) The shear strain associated with the transition is revealed by showing the relative position of oxygen atoms common to four unit cells in the cubic phase (schematized in grey) and for the orthorhombic phase (in red). The shear distortion is exaggerated by a factor of 300. Labels $a$ and $b$ correspond to the orthorhombic lattice parameters. Labelled angles correspond to the true values expected for the strained unit cell for $\mathrm{Mg}-\mathrm{Cl}$ boracite. (d) Local topographical map of $\mathrm{Cu}-\mathrm{Cl}$ boracite crystal surface as measured by atomic force microscopy. The scale bar measures $2 \mu \mathrm{m}$. (e) Corrected lateral-mode piezoresponse map (a.u.) for the area shown in $\mathbf{d}$ and scan obtained with relative cantilever orientation at $90^{\circ}$, (f). Grey motif indicates cantilever orientation. (g) Montage of piezoreponse maps in e,f. Coloured legend represents the four in-plane polarized domain states with orientations labelled by arrows.

crystal plate, measured using atomic force microscopy (AFM; Methods section), is shown in Fig. 1d. The observed surface deformations are consistent with the development of ferroelastic shear twins having boundaries with surface trace along $\langle 100\rangle_{\mathrm{pc}}$ and $\langle 110\rangle_{\mathrm{pc}}$ (ref. 26). Lateral-mode piezoresponse force microscopy (PFM; Methods section) of the same area in Fig. 1e-g sheds light on the polar domain structure; there is clearly spatial variation in the measured piezoresponse signals that correlates with the spatial distribution of ferroelastic domains. The piezoresponse maps presented in Fig. 1e,f have been corrected to remove a deflection-related cross-talk signal (due to low signal-to-noise associated with the genuine piezoresponse, see Supplementary Note 1). Combining the information in Fig. 1e,f into a single-domain map, the polarization of each domain can be assigned as shown in Fig. 1g. All the topographical boundaries from Fig. 1d can be identified in this image, indicating that the surface topography delineates the polar domain structure as well as the ferroelastic domains (as expected in boracites ${ }^{24}$ ). Vertical PFM maps show poor signal-to-noise with measured contrast that depends on sample orientation, suggesting that the signal is either flexural in origin and/or heavily influenced by deflection-related crosstalk. We therefore expect that all polarization lies in the plane parallel to the sample surface.

Nanoscale spatially-resolved current mapping (described in Methods section) is shown in Fig. 2a for the polydomain region imaged in Fig. 1d-g. Specific ferroelectric/ferroelastic domain walls (identified in Fig. 2b schematic) are seen to exhibit enhanced conductivity compared to the bulk. Also visible, but much fainter in signal, are boundaries that appear more resistive than the bulk. These measurements support Schmid and Pétermann's early speculation ${ }^{27}$, based on indirect bulk conductivity measurements, that domain walls in single crystal $\mathrm{Cu}-\mathrm{Cl}$ boracite may be conducting. Using the PFM analysis of Fig. 1, we can examine the polar structure, schematized in Fig. 2b, allowing us to identify head-to-head $90^{\circ}$ domain walls, tail-to-tail $90^{\circ}$ walls and uncharged $180^{\circ}$ domain walls. Schematic illustrations of how the sheared unit cell leads to the observed domain wall orientations and characteristic surface distortions are 
a

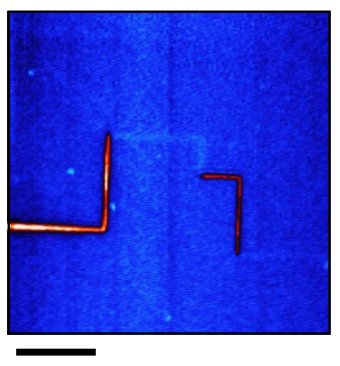

b

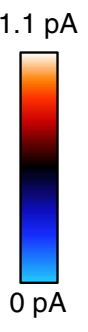

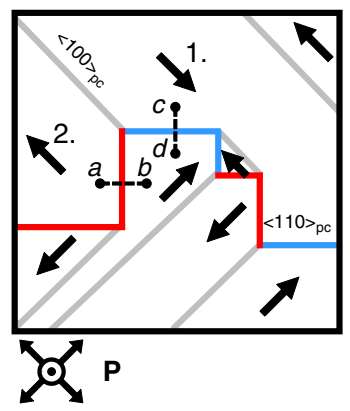

c

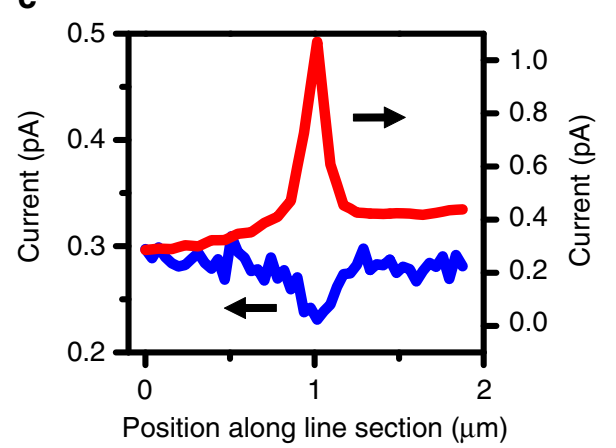

Figure 2 | Transport along charged walls. (a) Spatially resolved current map captured with $-12 \mathrm{~V}_{\mathrm{dc}}$ applied to the bottom electrode. The bulk background signal (order of $0.1 \mathrm{pA}$ ) has been removed to enhance wall-specific contrast. The scale bar measures $2 \mu \mathrm{m}$. (b) Schematic of polar domain structure with $90^{\circ}$ head-to-head and tail-to-tail charged boundaries coloured in blue and red respectively. Uncharged $180^{\circ}$ walls are indicated as grey lines. (c) Current line-sections taken across the conducting and insulating walls seen in $\mathbf{a}$. The conducting wall line profile (red) is taken across the dashed line path $a$ to $b$ in $\mathbf{b}$ while the insulating wall profile is taken along the path $c$ to $d$.

shown in Supplementary Figs 5 and 6. Comparison with the current map in Fig. 2a immediately reveals a direct correlation between the predicted charge-state of each wall and the local current amplitude experimentally measured. Tail-tail charged $90^{\circ}$ walls are seen to be solely responsible for the enhanced conduction and head-head charged $90^{\circ}$ walls account for the insulating walls. The observation that uncharged $180^{\circ}$ domain walls exhibit no current anomaly is further strong evidence that it is the charge state of the boundary that underpins the augmented or diminished local transport. The spontaneous development of charged walls in boracites is not surprising, as Schmid and coworkers have previously determined that the only mechanically compatible ferroelastic domain walls with composition plane $\{110\}_{\mathrm{pc}}$ correspond to the case of $90^{\circ}$ charged walls, as seen in our experiments ${ }^{24,25}$. Knowing this, we can determine the domain polarization map (to within a $180^{\circ}$ rotation of all polarization vectors) using solely the sheared domain topography as measured by atomic force microscopy (see Supplementary Note 2). We note that observation of conductivity enhancement/suppression being directly correlated with predicted charged-wall species is entirely consistent with local domain wall transport measurements made in other improper-ferroelectric crystals to date $\left(\mathrm{ErMnO}_{3}\right.$ (ref. 2) and in $(\mathrm{Ca}, \mathrm{Sr})_{3} \mathrm{Ti}_{2} \mathrm{O}_{7}$ (ref. 11)) as well as in proper ferroelectric $\mathrm{BaTiO}_{3}$ (ref. 10). We suggest that conduction in boracites is mediated by $p$-type majority carriers based on bulk Hall effect measurements (see Supplementary Note 3). The observation of tail-tail conducting walls here is therefore consistent with the prevailing theory that enhanced conductivity is observed along walls that are screened by majority-type carriers ${ }^{2,28}$.

Pressure writing of charged domain walls. We also demonstrate a technique for creating ordered patterns of these conducting domain walls using mechanical pressure, shown in Fig. 3. First, the crystal is heated to one degree below the phase transition $\left(T_{\mathrm{c}}=91^{\circ} \mathrm{C}\right.$ (ref. 29)) such that domain wall mobility is increased compared to room temperature and ferroelastic transformation plasticity developed. Next, a fine-tipped metal probe is pressed into the surface of the crystal. With applied pressure of order $1 \mathrm{GPa}$, dramatic domain reconfiguration is observed to occur around the contact point, up to hundreds of microns away (Fig. 3d), similar to that seen in nanoindentation experiments carried out on ferroelectrics ${ }^{30}$. The temperature is then reduced and the surface stress removed, leaving a depression formed by quadrants consisting of finer-scale domains. The pressure required to write this pattern is larger than the uniaxial pressure of $120 \mathrm{MPa}$ reported by Torre et al. ${ }^{31}$ required for room temperature ferroelastic domain switching in $\mathrm{Mg}-\mathrm{Cl}$ boracite; we expect our $1 \mathrm{GPa}$ estimate to be an upper limit since the magnitude of applied stress will decrease away from the point of contact (as opposed to uniform stress applied in the study by Torre et al. $^{31}$ ). Current mapping (Fig. 3b,c) shows that two of the written quadrant boundaries consist of conducting domain wall sections that extend in the same direction for hundreds of microns in length (Fig. 3d). Artificially written conducting walls can be over $50 \mu \mathrm{m}$ in length, as shown in Fig. 3b, and on occasion can be hundreds of microns long. The boundaries have a surface trace along $\langle 110\rangle_{\mathrm{pc}}$ indicating that the observed conductivity enhancement is consistent with the $90^{\circ}$ charged-wall interpretation described above. The other two opposing quadrant boundaries that trace along $\langle 110\rangle_{\mathrm{pc}}$ directions are therefore expected to have an in-plane polar discontinuity of the opposite charge sense (made clear in the schematic in Fig. 3e). Indeed, close examination of the current profile reveals segments that are more resistive than the bulk (Fig. 3c), consistent with the charged-wall picture. From a thermodynamics perspective, the specific quadrant pattern of ferroelastic domains can be rationalized to occur since they facilitate a depression of the surface in the immediate vicinity of the applied pressure. The strict orientational relationship between the structural shearing and polarization allows us to identify that domains which generate surface corrugation have polarization that is restricted to being in-plane and oriented perpendicular to the shear gradient. These mechanically written conducting wall patterns appear stable, showing no discernible change after almost 2 weeks and the writing process is seen to cause no damage in the vicinity of the probe-contact.

An important feature of this quadrant pattern with conducting domain walls along $\{110\}_{\mathrm{pc}}$ is that it can be formed reliably and site-specifically when this heat-assisted stress poling procedure is repeated. The pattern can be then erased by thermal-cycling through the modest phase transition temperature, annihilating the conducting walls. Using the approach pioneered by Gruverman and co-workers ${ }^{32}$, basic prototype devices that are actuated by nanoprobe-induced stress may be a possibility if boracite thin-films can be fabricated (to date, only Schmid et al. ${ }^{33}$ have attempted film growth). Some degree of scope for tuning the transport properties of the boracite system may be anticipated by appropriate choice of the metal-halogen combination. It should also be possible to write these charged domain wall patterns with point-like stress in other $\langle 100\rangle_{\mathrm{pc}}$-faced orthorhombic boracites since they should exhibit the same geometric shear response and therefore the same charged polar configuration. In fact, a 

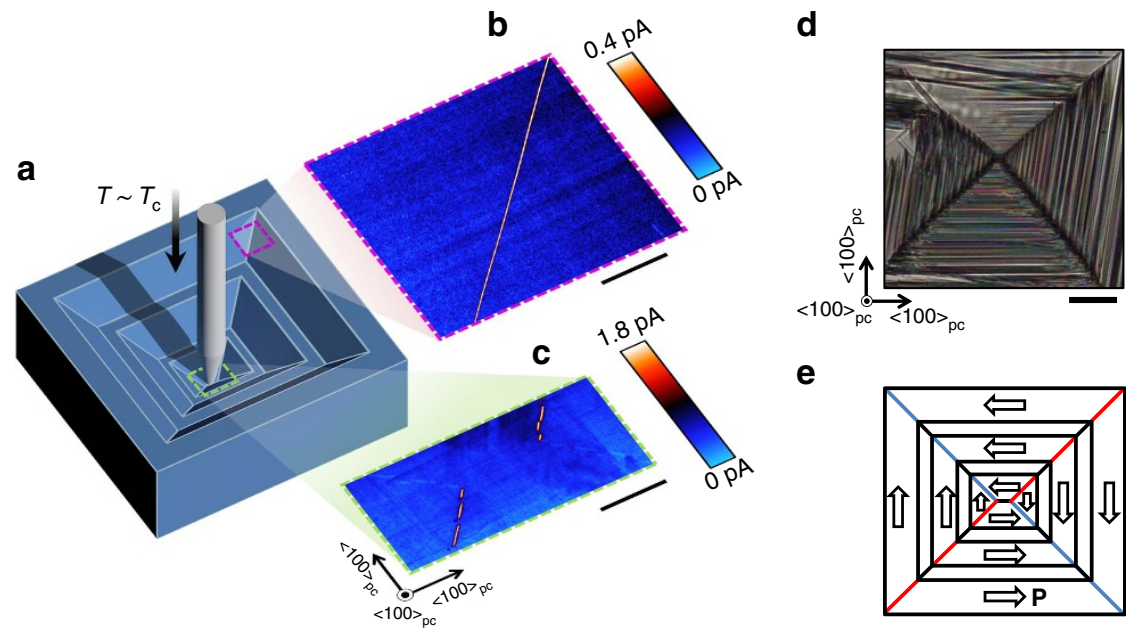

e

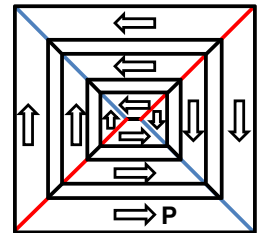

Figure 3 | Stress-field injection of charged domain walls near the Curie temperature. (a) Schematic quadrant domain structure after probe-applied stress at elevated temperature. Spatially resolved current map of an injected long conducting boundary, (b), and the distribution of conducting and insulating boundaries surrounding the point where stress is applied, (c). A bias of magnitude $-12 V_{d c}$ is applied to the bottom electrode. Differences in measured current values between panels $(\mathbf{b}, \mathbf{c})$ are due to wear of the conductive tip-coating rather than differences in the intrinsic transport properties of the boundary. The scale bars in $(\mathbf{b}, \mathbf{c})$ measure $10 \mu \mathrm{m}$. (d) Polarized light microscopy of the quadrant domain microstructure that develops around the point where probe-pressure is applied. (e) Schematic illustration of domain structure in $\mathbf{d}$ with in-plane domain polarizations indicated. Insulating head-to-head charged $90^{\circ}$ walls are indicated with blue lines and conducting tail-to-tail $90^{\circ}$ charged walls are indicated with red lines. Uncharged boundaries are indicated with black lines. The scale bar measures $100 \mu \mathrm{m}$.

a

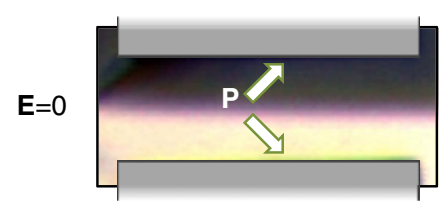

C

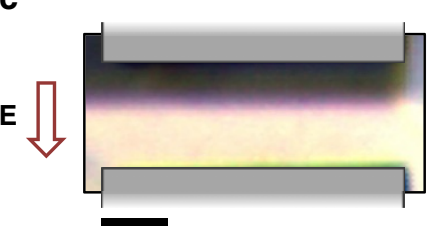

b

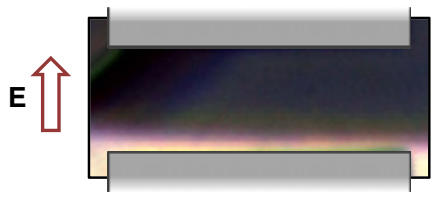

d

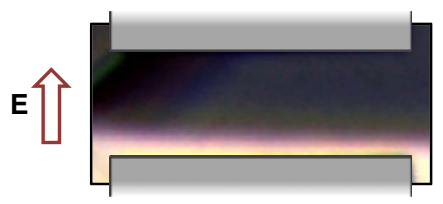

e

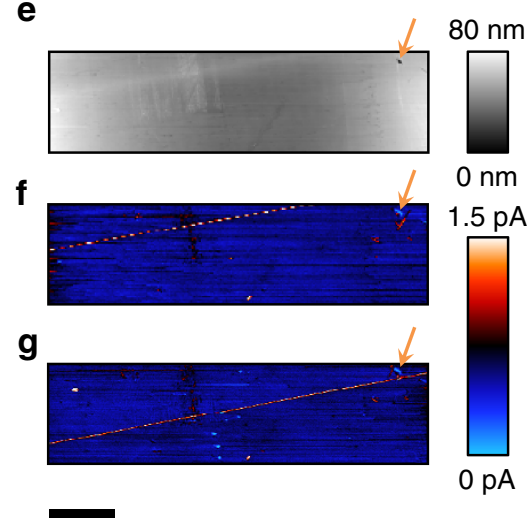

Figure 4 | Electric field control of conducting domain walls near the Curie temperature. (a) Polarized light microscopy of a conducting domain wall under zero applied field (E). (b) E-field applied between surface planar electrodes (schematized in grey) in positive sense causes wall to move towards bottom electrode. Arrows labelled $\mathbf{P}$ denote domain polarization direction. (c) Reversing applied field direction causes the wall to move towards the top electrode. (d) Field applied again in positive direction causes wall to move back towards the bottom electrode. The scale bar for a-d measures $25 \mu \mathrm{m}$. Topography, (e) and spatially resolved current map (f) of the conducting wall shown in a before switching fields are applied. (g) Spatially resolved current map of the conducting wall after switching shows that its transport properties are not changed by wall movement. The orange arrow points to a topographical marker which serves as a reference for relative wall position. The apparent 'beaded' profile of the conducting wall is an imaging artefact due to the cantilever scan axis being at an oblique angle to the wall. For nanoscale current imaging, a bias of $-10 V_{d c}$ is applied to the bottom electrode. The scale bar for $\mathbf{e}-\mathbf{g}$ measures $10 \mu \mathrm{m}$.

strikingly similar charged-wall configuration and quadrant microstructure has even been seen to form naturally in $\mathrm{Mg}_{3} \mathrm{~B}_{7} \mathrm{O}_{13} \mathrm{Cl}$ boracite crystals due to environmental stresses associated with their petrogenesis ${ }^{25,34}$.

Electric field control of charged domain wall position. We are also able to experimentally demonstrate that these stress-written walls can be moved using applied electric fields, as shown in Fig. 4a-d. Gold planar electrodes were deposited on the surface and the sample was heated to $90^{\circ} \mathrm{C}$ to increase charged-wall mobility. Using polarized light microscopy, we observed wall motion for electric field application of $30 \mathrm{kVcm}^{-1}$ and above (electric field-oriented in-plane and perpendicular to the wall) Reversing the electric field caused the charged wall to move in the opposite direction, demonstrating that the position of these walls can be well controlled using electric field manipulation, after being created using applied stress. Crucially for applications, local current mapping (Fig. 4e-g), carried out both before and after switching, shows that moving the charged wall with electric field does not adversely affect its transport properties.

\section{Discussion}

In summary, we have reported the first direct observation, controlled injection and field-controlled movement of conducting 
domain wall patterns in $\mathrm{Cu}-\mathrm{Cl}$ boracite. Such domain walls are straight, can be tens or even hundreds of microns long and the observed conduction variations correlate directly with the charge state of the wall. We have demonstrated a simple site-specific domain wall writing technique using locally applied stress to reliably trigger growth of conducting domain wall patterns that span up to hundreds of microns in length. Furthermore, these walls can be repositioned using applied electric fields, an important requirement for conventional voltage-operated devices. For future work, parallel studies should be carried out on thin-film thicknesses of boracite material with highly localized pressure exerted using an atomic force microscope probe (such as in ref. 35). In particular, the density of domain walls injected using a local probe should be mapped fully as a function of temperature and pressure to explore the parameter space for which these conducting walls are stabilized.

\section{Methods}

Sample preparation and atomistic structure models. Single crystals of $\mathrm{Cu}_{3} \mathrm{~B}_{7} \mathrm{O}_{13} \mathrm{Cl}$, several millimetres in size, have been grown using a sealed ampoule, vapour phase transport technique ${ }^{36}$. Approximately $0.5 \mathrm{~mm}$ thick $(100)_{\mathrm{pc}}$-faced single crystals plates were obtained from the parent crystal by diamond sawing followed by a chemical-mechanical polish process. $\mathrm{Cu}-\mathrm{Cl}$ boracite single crystals have a reported spontaneous polarization of $\sim 1.8 \mu \mathrm{C} \mathrm{cm}^{-2}$ and a coercive-field of up to $50 \mathrm{kV} \mathrm{cm}^{-1}$ as measured by Schmid et al..$^{37}$. CrystalMaker software was used to make the atomistic models shown in Fig. 1 with atomic coordinates for $\mathrm{Mg}-\mathrm{Cl}$ boracite as an input from the study by Ito et al. ${ }^{22}$.

Scanning probe microscopy characterization. AFM topography mapping is carried out using a Veeco Dimension 3100 AFM system with a Nanoscope IIIa controller. PFM measurements are carried out using the same AFM system in conjunction with an EG\&G 7265 lock-in amplifier. Commercially obtained $\mathrm{Pt} / \mathrm{Ir}$-coated Si probes are used (Nanosensors model PPP-EFM) with a probing signal of $5 \mathrm{~V}_{\mathrm{ac}}$ at $20 \mathrm{kHz}$. Spatially resolved current mapping is carried out at room temperature using the AFM system in conjunction with a Bruker Tunnelling AFM module. Voltages up to $-12 \mathrm{~V}_{\mathrm{dc}}$ are applied to the base of the crystal while the tip is grounded.

Electric field-driven motion of charged domain walls. For electric fieldswitching experiments, $100 \mathrm{~nm}$ of $\mathrm{Au}$ is sputter-deposited through a patterned transmission electron microscopy grid on top of the stress-written conducting domain wall pattern resulting in an array of $100 \times 100 \mu \mathrm{m}^{2}$ square-shaped surface electrodes. The crystal is then suspended across a hole in the centre of a ceramic heating element to allow for simultaneous heating (with temperature monitored by a thermocouple connected to a Thorlabs Model TC200 Controller) while transmission optical microscopy was carried out. A pair of electrodes located either side of the conducting wall are biased up to $\pm 200 \mathrm{~V}_{\mathrm{dc}}$ using a Keithley Model 237 source/measure unit and the charged domain wall position is then recorded using polarized light microscopy.

Data availability. All data used to support the findings of this study are available from the corresponding author upon request.

\section{References}

1. Schröder, M. et al. Conducting domain walls in lithium niobate single crystals. Adv. Func. Mater. 22, 3936-3944 (2012).

2. Meier, D. et al. Anisotropic conductance at improper ferroelectric domain walls. Nat. Mater. 11, 284-288 (2012).

3. Maksymovych, P. et al. Tunable metallic conductance in ferroelectric nanodomains. Nano Lett. 12, 209-213 (2011).

4. Aird, A. \& Salje, E. K. H. Sheet superconductivity in twin walls: experimental evidence of. J. Phys. Condens. Matter 10, L377-L380 (1998).

5. Seidel, J. et al. Conduction at domain walls in oxide multiferroics. Nat. Mater. 8, 229-234 (2009).

6. Seidel, J. et al. Domain wall conductivity in La-doped $\mathrm{BiFeO}_{3}$. Phys. Rev. Lett. 105, 197603 (2010).

7. Schaab, J. et al. Optimization of electronic domain-wall properties by aliovalent cation substitution. Adv. Electron. Mater. 2, 1500195 (2016).

8. Guyonnet, J., Gaponenko, I., Gariglio, S. \& Paruch, P. Conduction at domain walls in insulating $\mathrm{Pb}\left(\mathrm{Zr}_{0.2} \mathrm{Ti}_{0.8}\right) \mathrm{O}_{3}$ thin films. Adv. Mater. 23, 5377-5382 (2011).

9. Farokhipoor, S. \& Noheda, B. Conduction through $71^{\circ}$ domain walls in $\mathrm{BiFeO}_{3}$ thin films. Phys. Rev. Lett. 107, 127601 (2011).
10. Sluka, T., Tagantsev, A. K., Bednyakov, P. \& Setter, N. Free-electron gas at charged domain walls in insulating $\mathrm{BaTiO}_{3}$. Nat. Commun. 4, 1808 (2013).

11. Oh, Y. S., Luo, X., Huang, F.-T., Wang, Y. \& Cheong, S.-W. Experimental demonstration of hybrid improper ferroelectricity and the presence of abundant charged walls in $(\mathrm{Ca}, \mathrm{Sr})_{3} \mathrm{Ti}_{2} \mathrm{O}_{7}$ crystals. Nat. Mater. 14, 407-413 (2015).

12. Crassous, A., Sluka, T., Tagantsev, A. K. \& Setter, N. Polarization charge as a reconfigurable quasi-dopant in ferroelectric thin films. Nat. Nanotech. 10, 614-618 (2015).

13. Tagantsev, A. K., Cross, L. E. \& Fousek, J. Domains in Ferroelectric Crystals and Thin Films 70-71 (Springer, 2010).

14. Levanyuk, A. P. \& Sannikov, D. G. Improper ferroelectrics. Sov. Phys. Usp. 17, 199-214 (1974).

15. Lilienblum, M. et al. Ferroelectricity in the multiferroic hexagonal manganites. Nat. Phys. 11, 1070-1073 (2015)

16. Fousek, J. \& Janovec, V. The orientation of domain walls in twinned ferroelectric crystals. J. Appl. Phys. 40, 135-142 (1969).

17. Keve, E. T., Abrahams, S. C., Nassau, K. \& Glass, A. M. Ferroelectric ferroelastic paramagnetic terbium molybdate $\beta-\mathrm{Tb}_{2}\left(\mathrm{MoO}_{4}\right)_{3}$. Solid State Commun. 8 , 1517-1520 (1970).

18. Erhart, J. Domain wall orientations in ferroelastics and ferroelectrics. Phase Transit. 77, 989-1074 (2004).

19. Bednyakov, P., Sluka, T., Tagantsev, A., Damjanovic, D. \& Setter, N. Free-carrier-compensated charged domain walls produced with super-bandgap illumination in insulating ferroelectrics. Adv. Mater. 28, 9498-9503 (2016).

20. Solomon, P. M. et al. Pathway to the piezoelectronic transduction logic device. Nano Lett. 15, 2391-2395 (2015).

21. Fouassier, C., Levasseur, A., Joubert, J. C., Muller, J. \& Hagenmuller, P. Les Systèmes $\mathrm{B}_{2} \mathrm{O}_{3}$-MO-MS boracites $\mathrm{M}-\mathrm{S}(\mathrm{M}=\mathrm{Mg}, \mathrm{Mn}, \mathrm{Fe}, \mathrm{Cd})$ et sodalites M-S $(\mathrm{M}=\mathrm{Co}, \mathrm{Zn})$. Z. Anorg. Allg. Chem. 375, 202-208 (1970).

22. Ito, T., Morimoto, N. \& Sadanaga, R. The crystal structure of boracite. Acta Cryst. 4, 310-316 (1951).

23. Thornley, F. R., Nelmes, R. J. \& Kennedy, N. S. J. Structural studies of Cu-Cl-boracite. Ferroelectrics 13, 357-359 (1976).

24. Zimmermann, A., Bollmann, W. \& Schmid, H. Observations of ferroelectric domains in boracites. Phys. Status Solidi A 3, 707-720 (1970).

25. Schmid, H. in Growth of Crystals Volume 7 (eds Shubnikova, A. V. \& Sheftal, N. N.) 25-52 (Consultants Bureau, 1969).

26. Ye, Z.-G., Janner, A.-M. \& Schmid, H. Structural and magnetic phase transitions in Fe-I boracite. J. Phys. Condens. Matter 9, 2607-2621 (1997).

27. Schmid, H. \& Pétermann, L. A. Dielectric constant and electric resistivity of copper chlorine boracite, $\mathrm{Cu}_{3} \mathrm{~B}_{7} \mathrm{O}_{13} \mathrm{Cl}(\mathrm{Cu}-\mathrm{Cl}-\mathrm{B})$. Phys. Status Solidi A 41, K147-K150 (1977).

28. Eliseev, E. A., Morozovska, A. N., Svechnikov, G. S., Gopalan, V. \& Shur, V. Y. Static conductivity of charged domain walls in uniaxial ferroelectric semiconductors. Phys. Rev. B 83, 235313 (2011).

29. Ascher, E., Schmid, H. \& Tar, D. Dielectric properties of boracites and evidence for ferroelectricity. Solid State Commun. 2, 45-49 (1964).

30. Schneider, G. A., Scholz, T., Muñoz-Saldaña, J. \& Swain, M. V. Domain rearrangement during nanoindentation in single-crystalline barium titanate measured by atomic force microscopy and piezoresponse force microscopy. Appl. Phys. Lett. 86, 192903 (2005).

31. Torre, L. P., Abrahams, S. C. \& Barns, R. L. Ferroelectric and ferroelastic properties of Mg-Cl-Boracite. Ferroelectrics 4, 291-297 (1972).

32. Lu, H. et al. Mechanical writing of ferroelectric polarisation. Science 336, 59-61 (2012).

33. Schmid, H. \& Tippman, H. Gas phase synthesis of epitaxial layers of nickel-chlorine boracite on chromium-chlorine boracite. J. Cryst. Growth 46, 723-742 (1979).

34. Mack, K. Ueber das pyroelektrische Verhalten des Boracits. Z. Kristallogr. 8, 503-522 (1884).

35. Stolichnov, I. et al. Bent ferroelectric domain walls as reconfigurable metallic-like channels. Nano Lett. 24, 8049-8055 (2015).

36. Whatmore, R. W., Brierley, C. J. \& Ainger, F. W. Nucleation control during the growth of boracite single-crystals. Ferroelectrics 28, 329-332 (1980).

37. Schmid, H., Genequand, P., Pouilly, G. \& Chan, P. Pyroelectricity of Fe-I and $\mathrm{Cu}-\mathrm{Cl}$ boracite. Ferroelectrics 25, 539-542 (1980).

\section{Acknowledgements}

We acknowledge financial support from the Engineering and Physical Sciences Research Council (EPSRC) Grant No. EP/J017191/1 and EP/N018389/1. M.P.C. and J.M.G. acknowledge support through Department of Employment and Learning (DEL). We acknowledge insightful discussions with Dr A. Morelli and the late Prof H. Schmid. The assistance of $\mathrm{Mr} \mathrm{C}$. Brierley in growing the crystal used is gratefully acknowledged. We are grateful to Dr M. Arredondo and Mr M. O'Leary for transmission electron microscopy of the crystals to confirm crystal orientation. 


\section{Author contributions}

R.W.W. suggested boracites for interesting domain wall behaviour. R.G.P.McQ., A.K. and J.M.G. conceived of and designed the subsequent experiments. R.G.P.McQ., M.P.C. and A.K. performed the experiments described and analysed the associated data; R.W.W. made and provided the boracite single crystal material; J.M.G. supervised the research and helped in data analysis and interpretation. R.G.P.McQ. and J.M.G. primarily wrote the manuscript with input from all authors.

\section{Additional information}

Supplementary Information accompanies this paper at http://www.nature.com/ naturecommunications

Competing interests: The authors declare no competing financial interests.

Reprints and permission information is available online at http://npg.nature.com/ reprintsandpermissions/
How to cite this article: McQuaid, R. G. P. et al. Injection and controlled motion of conducting domain walls in improper ferroelectric $\mathrm{Cu}-\mathrm{Cl}$ boracite. Nat. Commun. 8, 15105 doi: 10.1038/ncomms15105 (2017).

Publisher's note: Springer Nature remains neutral with regard to jurisdictional claims in published maps and institutional affiliations.

(c) (i) This work is licensed under a Creative Commons Attribution 4.0 International License. The images or other third party material in this article are included in the article's Creative Commons license, unless indicated otherwise in the credit line; if the material is not included under the Creative Commons license, users will need to obtain permission from the license holder to reproduce the material. To view a copy of this license, visit http://creativecommons.org/licenses/by/4.0/

(C) The Author(s) 2017 